\title{
Takagi-Sugeno Fuzzy Observer Design for Induction Motors with Immeasurable Decision Variables: State Estimation and Sensor Fault Detection
}

\author{
M. Allouche, \\ M. Chaabane, \\ M. Souissi \\ Unit of Industrial Processes \\ Control, UCPI, National \\ Engineering School of Sfax, \\ ENIS, Department of \\ Electrical Engineering, BP \\ 1173, 3038 Sfax Tunisia
}

\author{
D. Mehdi \\ Laboratory of Automatic \\ Informatics Industrial, L.A.I.I, \\ Superior School of Engineers \\ of Poitiers, 40 Avenue du \\ Recteur Pineau, 86022 \\ Poitiers, France
}

\author{
F. Tadeo \\ Dpt. Systems Engineering, \\ Universidad de Valladolid, \\ 47011 Valladolid, Spain.
}

\begin{abstract}
This paper deals with the problem of sensor fault detection of induction motors described by some linear models blended together through non linear membership functions that involve unmeasurable decision variables. The intermittent disconnections of the sensors produce severe transient errors in the estimator used in the control loop, worsening the performance of the induction motor. Then, a Takagi-Sugeno (TS) observer is proposed, in descriptor form, to simultaneously estimate the states and achieve the detection and isolation of incipient sensors faults. For this, a TS model is first derived to represent precisely the induction motor in the fixed stator $\mathrm{d}-\mathrm{q}$ reference frame. Secondly, a descriptor TS observer is synthesized, in which the sensor faults are considered as an auxiliary variable state. Some simulation results illustrate the effectiveness of the proposed approach
\end{abstract}

\section{General Terms}

Observer design, intermittent disconnections,

\section{Keywords}

Induction motor, sensor faults, Takagi-Sugeno models, fuzzy descriptor observer, Linear Matrix Inequalities (LMIs).

\section{INTRODUCTION}

Three-phase induction motors are very frequent in industrial processes. This is mainly due to their low cost, reasonable size, ruggedness and low maintenance requirements. Usually, these motors are equipped with sensors for current and rotor speed that work under many stresses from different origins (thermal, electric, mechanical and environment), which seriously affect the lifespan of the sensors, creating sensor faults. These sensors are normally optical encoders and Hall-effect current sensors: they occasionally fail, and their resulting accidental downtime can be very expensive, due to the reduction of the production quality. Moreover, they create serious safety hazards for the operators. Hence, the detection and isolation of sensor faults becomes a necessary task, since control systems rely on the information provided by the signals generated by these sensors. Right now, sensor faults are treated in practice by using filters in the control system, but this solution does not completely eliminate the faults and often implies the degradation of controller performance. Thus, considerable research is being devoted to providing techniques for sensor fault detection and diagnosis [1, 2, 3, 4, 5]. Among these techniques, the methods based on the analysis of stator currents and vibration signals are the best-known, as they are based on simple measurements and are highly reliable [2]. However, these diagnostic techniques are specific for fixed frequency PWM drives, whereas using a variable-frequency PWM drive, as it is frequent in practice, would make the accurate detection of spectral components from faults very difficult.

Recently, substantial research efforts have been devoted to methods based on artificial intelligence techniques, such as neural networks and fuzzy logic, to deal with the problems of diagnosis and fault tolerant control applied to induction motors $[6,7,8,9,10,11,12]$. Much research on this issue is carried out using Takagi-Sugeno (TS) models [6, 7]. This TS fuzzy approach has been extensively used to model nonlinear systems and has been successfully applied to practical problems [20]. The basic idea for the approach is to decompose the model of a nonlinear system into a set of linear subsystems with associated nonlinear weighting functions.

In this context, and using the TS approach, the methodologies presented in $[6,7]$ to deal with the problem of fault diagnostics consider that the weighting functions depend on measurable decision variables. Indeed, based on the bilinear matrix modeling and using the Takagi-Sugeno (TS) approach, Bennett et al. proposed a Generalized Observer Scheme in [6] to detect and isolate sensor faults, which was successfully applied to an induction motor traction system. The basic idea was to use a bank of TS observers to generate residuals for each monitored system output signal. However, these observers provide large error estimation when a sensor disconnection takes place and, as a result, it becomes hard to detect and locate the faults perfectly. For this reason, it is relevant to consider the case where the unmeasurable variables defined by the rotor flux are used as premise variables, so the observer will not be sensitive to the intermittent disconnections that affect the sensors.

Thus, this paper studies the TS fault estimation of induction motor sensors with unmeasurable decision variables. The sensor faults considered in this work are disconnections: these are large faults, but may only exist for very short durations. To develop the technique, we first provide a representation of the dynamic 
behavior of induction motors by the nonlinear interpolation of a set of linear sub-models, thus providing a TS model. The second step is to develop a TS observer, to simultaneously estimate the system states and the fault signal by using the known input $\mathrm{u}(\mathrm{t})$ and the measurement output $y(t)$. The proposed methodology is applicable in similar engineering problems, as long as a good (nonlinear) model of the system is available. Compared with other fuzzy design methodologies, the TS fuzzy approach is directly based on pre-existing physical models, and using the LMI tools it's possible to verify the global stability of the fuzzy observer, even in the presence of sensor faults, so it is appealing for industrial application. Moreover and compared with other nonlinear techniques of diagnostic based on the use of a banc of observers, the designed descriptor observer is simple to be implemented.

\section{PRELIMINARIES}

\subsection{Physical model of induction motor}

Under the assumptions of linearity of the magnetic circuit, the dynamic model of the induction motor in the fixed stator $\mathrm{d}-\mathrm{q}$ reference frame can be described as follows (see, for example, $[18,22])$ :

$$
x(t)=f(x(t))+g(x(t)) u(t)+w(t)
$$

Where

$$
\begin{aligned}
& f(x(t))=\left[\begin{array}{c}
-\gamma i_{s d}+\frac{K_{s}}{\tau_{r}} \Psi_{r d}+K_{s} n_{p} \omega_{m} \Psi_{r q} \\
-\gamma i_{s q}-K_{s} n_{p} \omega_{m} \Psi_{r d}+\frac{K_{s}}{\tau_{r}} \Psi_{r q} \\
\frac{M}{\tau_{r}} i_{s d}-\frac{1}{\tau_{r}} \Psi_{r d}-n_{p} \omega_{m} \Psi_{r q} \\
\frac{M}{\tau_{r}} i_{s q}+n_{p} \omega_{m} \Psi_{r d}-\frac{1}{\tau_{r}} \Psi_{r q} \\
\frac{n_{p} M}{J L_{r}}\left(\Psi_{r d} i_{s q}-\Psi_{r q} i_{s d}\right)-\frac{f}{J} w_{m}
\end{array}\right], g(x(t))=\left[\begin{array}{cc}
\frac{1}{\sigma L_{s}} & 0 \\
0 & \frac{1}{\sigma L_{s}} \\
0 & 0 \\
0 & 0 \\
0 & 0
\end{array}\right] \\
& x(t)=\left[\begin{array}{lllll}
i_{s d} & i_{s q} & \Psi_{r d} & \Psi_{r q} & \omega_{m}
\end{array}\right]^{T} u(t)=\left[\begin{array}{ll}
u_{s d} & u_{s q}
\end{array}\right]^{T},
\end{aligned}
$$

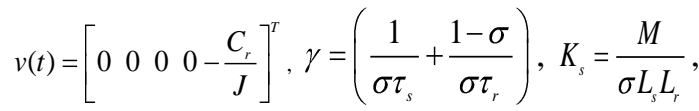

$$
\begin{aligned}
& \tau_{r}=\frac{1}{\alpha}=\frac{L_{r}}{R_{r}} \tau_{s}=\frac{L_{s}}{R_{s}}, \sigma=1-\frac{M^{2}}{L_{s} L_{r}}, \mu=\frac{3 n_{p} M}{2 J L_{r}}
\end{aligned}
$$

In which $\omega_{m}$ is the rotor speed, $\left(\Psi_{r d}, \Psi_{r q}\right)$ are the rotor fluxes, $\left(i_{s d}, i_{s q}\right)$ are the stator currents and $\left(u_{s d}, u_{s q}\right)$ are the stator voltages. The load torque $C_{r}$ is an exogenous disturbance. The motor parameters are: The moment of inertia $J$, the rotor and stator resistances $\left(R_{s}, R_{r}\right)$, the inductances $\left(L_{s}, L_{r}\right)$, the mutual inductance $M$, the friction coefficient $f$ and the number of poles pairs $n_{p}$.

\subsection{Feedback control of the induction motor}

A methodology for state feedback control is now presented witch uses the descriptor observer describes in section 3 to deal with the state estimation of the control system. In the induction motor, the outputs to be controlled are the motor speed $\omega_{m}$ and the square of the magnitude of the total rotor flux $\left(\Psi_{r d}^{2}+\Psi_{r q}^{2}\right)$. The input-output linearizing feedback control law proposed in this paragraph requires the design of fuzzy observer, to provide a precise estimation of the inaccessible state defined by the rotor flux. Therefore, let

$$
\begin{aligned}
h(x(t)) & =\left[\begin{array}{l}
h_{1}(x(t)) \\
h_{2}(x(t))
\end{array}\right] \\
& =\left[\begin{array}{c}
\omega_{m} \\
\left(\Psi_{r d}^{2}+\Psi_{r q}^{2}\right)
\end{array}\right]
\end{aligned}
$$

The motor model given by (1) can be converted into a linearizable feedback form via state-transformation (for details see [17]). The linearized dynamic model of the IM can be written as follows:

$\left[\begin{array}{l}\ddot{y}_{1} \\ \ddot{y}_{2}\end{array}\right]=\left[\begin{array}{l}L_{f}^{2} h_{1} \\ L_{f}^{2} h_{2}\end{array}\right]+\beta(x)\left[\begin{array}{l}u_{s d} \\ u_{s q}\end{array}\right]$

where $\beta(x)$ is the decoupling matrix defined as

$$
\beta(x)=\left[\begin{array}{cc}
-\frac{\mu}{\sigma L_{s}} \Psi_{r q} & \frac{\mu}{\sigma L_{s}} \Psi_{r d} \\
\frac{2 \alpha M}{\sigma L_{s}} \Psi_{r d} & \frac{2 \alpha M}{\sigma L_{s}} \Psi_{r q}
\end{array}\right]
$$

The quantities to be controlled are differentiated with respect to time until the input control signals $u_{s d}$ and $u_{s q}$ appear and the derivatives of the state variables are eliminated. The terms $L_{f}^{2} h_{1}$ and $L_{f}^{2} h_{2}$ are the second Lie derivatives of $h(x)$ along a vector field $f(x(t))$, which are given by the following expressions:

$$
\begin{aligned}
L_{f}^{2} h_{1}(x)= & -\mu K_{s} n_{p} \omega_{m}\left(\Psi_{r d}^{2}+\Psi_{r q}^{2}\right)-\mu(\alpha+\gamma)\left(\Psi_{r d} i_{s q}-\Psi_{r q} i_{s d}\right) \\
& -\mu n_{p} \omega_{m}\left(\Psi_{r d} i_{s d}+\Psi_{r q} i_{s q}\right) \\
L_{f}^{2} h_{2}(x)= & \left(4 \alpha^{2}+2 \alpha^{2} K_{s} M\right)\left(\Psi_{r d}^{2}+\Psi_{r q}^{2}\right)+2 \alpha M n_{p} \omega_{m}\left(\Psi_{r d} i_{s q}-\Psi_{r q} i_{s d}\right) \\
& -\left(6 \alpha^{2} M+2 \alpha \gamma M\right)\left(\Psi_{r d} i_{s d}+\Psi_{r q} i_{s q}\right)+2 \alpha^{2} M^{2}\left(i_{s d}^{2}+i_{s q}^{2}\right)
\end{aligned}
$$

Since $\beta(x)=-\frac{2 \alpha M \mu}{\left(\sigma L_{s}\right)^{2}}\left(\Psi_{r d}^{2}+\Psi_{r q}^{2}\right) \neq 0$, then $\beta(x)$ is nonsingular, so the induction motor is input-output linearizable using the following control law:

$\left(\begin{array}{l}u_{s d} \\ u_{s q}\end{array}\right)=\beta^{-1}(x)\left(\left[\begin{array}{l}L_{f}^{2} h_{1}(x) \\ L_{f}^{2} h_{2}(x)\end{array}\right]+\left[\begin{array}{l}v_{a} \\ v_{b}\end{array}\right]\right)$ 
where $v=v_{a} \quad v_{b}{ }^{T}$ is the new input vector.

To ensure perfect tracking of the rotor speed $\omega_{m}$ and the square of the rotor flux norm $\left|\Psi_{r}\right|^{2}=\left(\Psi_{r d}^{2}+\Psi_{r q}^{2}\right), \quad v_{a}$ and $v_{b}$ are chosen as follows:

$v_{a}=-k_{a 1}\left(\omega_{m}-\omega_{m_{r e f}}\right)-k_{a 2}\left(\dot{\omega}_{m}-\dot{\omega}_{m_{r e f}}\right)+\ddot{\omega}_{m_{r e f}}$,

$v_{b}=-k_{b 1}\left|\Psi_{r}\right|^{2}-\left|\Psi_{r}\right|_{r e f}^{2}-k_{b 2}\left|\dot{\Psi}_{r}\right|^{2}-\left|\dot{\Psi}_{r}\right|_{r e f}^{2}+\left|\ddot{\Psi}_{r}\right|_{r e f}^{2}$,

where $\left(k_{a 1}, k_{a 2}\right)$ and $\left(k_{b 1}, k_{b 2}\right)$ are constant parameters

\section{TAKAGI-SUGENO MODELS OF INDUCTION MOTORS}

It is now shown that Takagi-Sugeno (TS) models make it possible to represent the behavior of the induction by the interpolation of a set of linear submodels [13, 14]. Each submodel contributes to the global behavior of the nonlinear system through a weighting function $h_{i}(z(t))$. These Takagi-Sugeno models are frequently used as an exact representation of nonlinear systems that allow the nonlinear behaviour of a system to be adequately reproduced using fuzzy combinations of local models. In fact in [21] it was shown how this type of models can be used to accurately represent a large class of nonlinear models (in a determined compact region). We must point out that in [6] a TS model of an induction motor was also derived, by using bilinear matrix modeling. In this work, an exact TS representation of the induction motor is obtained by transformation using the nonlinear sector approach.

First, a TS model of the induction motor is obtained, based on the measurable variables (currents and rotor speed). Later on, an alternative TS model is derived using the unmeasurable states as decision variables, as this makes possible to derive a more compact representation of the induction motor, as well as it has the advantage to be less sensitive to sensor faults (as measurements are not directly used as premise variables)

\subsection{TS model with measurable decision variables}

To express the model of the induction motor as a TS model with the measurable parameters (currents and rotor speed) as decision variables, we rewrite the equations (1) in the following equivalent state space form:

$\left\{\begin{array}{l}\square(t)=A(x) x(t)+B u(t)+D w(t) \\ y=C x(t)\end{array}\right.$

where:

$$
A(x(t))=\left[\begin{array}{ccccc}
-\gamma & 0 & \frac{K_{s}}{\tau_{r}} & K_{s} n_{p} \omega_{m} & 0 \\
0 & -\gamma & -K_{s} n_{p} \omega_{m} & \frac{K_{s}}{\tau_{r}} & 0 \\
\frac{M}{\tau_{r}} & 0 & -\frac{1}{\tau_{r}} & -n_{p} \omega_{m} & 0 \\
0 & \frac{M}{\tau_{r}} & n_{p} \omega_{m} & -\frac{1}{\tau_{r}} & 0 \\
0 & 0 & \mu i_{s q} & -\mu i_{s d} & -\frac{f}{J}
\end{array}\right], B=\left[\begin{array}{cc}
\frac{1}{\sigma L_{s}} & 0 \\
0 & \frac{1}{\sigma L_{s}} \\
0 & 0 \\
0 & 0 \\
0 & 0
\end{array}\right],
$$

$C=\left[\begin{array}{lllll}1 & 0 & 0 & 0 & 0 \\ 0 & 1 & 0 & 0 & 0 \\ 0 & 0 & 0 & 0 & 1\end{array}\right], \quad D=\left[\begin{array}{c}0 \\ 0 \\ 0 \\ 0 \\ 1 \\ -\frac{1}{J}\end{array}\right]$ and $w(t)=C_{r}$

Considering the sector of nonlinearities of the terms $z_{j}=x_{j} \in\left[z_{j \min }, z_{j \max }\right]$ of the matrix $A(x(t))$ with $j=1,2,3$ and the decision variables to be the measurable states:

$\left\{\begin{array}{l}z_{1}(t)=i_{s d}(t) \\ z_{2}(t)=i_{s q}(t) \\ z_{3}(t)=\omega_{m}(t)\end{array}\right.$

A convex polytypic transformation for these premise variables can be performed. For this, let us define:

$$
\left\{\begin{array}{l}
F_{j, \text { min }}\left(z_{j}\right)=\frac{z_{j}-z_{j \min }}{z_{j \max -} z_{j \min }} \\
F_{j, \text { max }}\left(z_{j}\right)=\frac{z_{j \max }-z_{j}}{z_{j \max -} z_{j \min }}
\end{array}\right.
$$

That fulfil the property $F_{j, \min }\left(z_{j}\right) .+F_{j, \max }\left(z_{j}\right)=1$.Then, the $j^{\text {th }}$ premise variable can be expressed as follows:

$$
z_{j}(t)=F_{j, \min }\left(z_{j}\right) z_{j \max }+F_{j, \max }\left(z_{j}\right) z_{j \min }
$$

Using this transformation, $r=2^{3}$ submodels are generated. The TS model is then described by a set of eight fuzzy If-Then rules, where the $i^{\text {th }}$ rule is defined as follows:

Rule $R i$

$$
\begin{gathered}
\text { If }\left(z_{1}(t) \text { is } F_{1, k}\right) \text { and }\left(z_{2}(t) \text { is } F_{2, l}\right) \text { and }\left(z_{3}(t) \text { is } F_{3, f}\right) \\
\text { Then } x(t)=A_{i} x(t)+B u(t)+D w(t),
\end{gathered}
$$

with $k, l, f \in \min , \max$ and $i=1,2, \ldots, 8$.

The global TS model with sensors faults $f_{s}(t)$ is then inferred as follows:

$\left\{\begin{array}{l}\dot{x}(t)=\sum_{i=1}^{8} h_{i}(x(t))\left(A_{i} x(t)\right)+B u(t)+D w(t) \\ y(t)=C x(t)+D_{s} f_{s}(t)\end{array}\right.$ 
where

$$
\begin{aligned}
& \lambda_{i}\left(z_{1}, z_{2}, z_{3}\right)=\prod_{k, l, f=1}^{2} F_{1}^{k}\left(z_{1}(t)\right) F_{2}^{l}\left(z_{2}(t)\right) F_{3}^{f}\left(z_{3}(t)\right) \\
& h_{i}(z(t))=\frac{\lambda_{i}\left(z_{1}, z_{2}, z_{3}\right)}{\sum_{i=1}^{8} \lambda_{i}\left(z_{1}, z_{2}, z_{3}\right)} \\
& \sum_{i=1}^{8} h_{i}(z(t))=1
\end{aligned}
$$

and $D_{s}$ is a matrix with appropriate dimensions

\subsection{TS model with unmeasurable decision variables}

The fuzzy TS model of the induction motors developed in the previous section is no longer valid for observer design when there are faults in the sensors. Thus, we now derive a new TS model for induction motors that uses only unmeasurable decision variables, so they are less affected by faults. Moreover, as only two variables are used, this makes possible to reduce the number of rules in the TS description of the system, which makes possible to obtain a more compact representation of the system.

Thus, selecting as premise variables of the TS model the two daxis and q-axis fluxes (i.e., $\Psi_{r d}(t)$ and $\Psi_{r q}(t)$ ), the induction motor can also be represented as the following TS model:

$$
\left\{\begin{array}{l}
x(t)=A(\hat{x}(t)) x(t)+B u(t)+D w(t) \\
y=C x(t)
\end{array}\right.
$$

where

$$
A(\hat{x}(t))=\left[\begin{array}{ccccc}
-\gamma & 0 & \frac{K_{s}}{\tau_{r}} & 0 & K_{s} n_{p} \Psi_{r q} \\
0 & -\gamma & 0 & \frac{K_{s}}{\tau_{r}} & -K_{s} n_{p} \Psi_{r d} \\
\frac{M}{\tau_{r}} & 0 & -\frac{1}{\tau_{r}} & 0 & -n_{p} \Psi_{r q} \\
0 & \frac{M}{\tau_{r}} & 0 & -\frac{1}{\tau_{r}} & n_{p} \Psi_{r d} \\
-\mu \Psi_{r q} & \mu \Psi_{r d} & 0 & 0 & -\frac{f}{J}
\end{array}\right]
$$

Considering the sector of nonlinearities of the terms $z_{j}=x_{j} \in\left[z_{j \min }, z_{j \max }\right] \quad$ of the matrix $A(\hat{x}(t))$ with $j=1,2$ and the premise variables the two unmeasurable states:

$\left\{\begin{array}{l}z_{1}(t)=\Psi_{r d}(t) \\ z_{2}(t)=\Psi_{r q}(t)\end{array}\right.$

makes possible to perform a convex polytypic transformation for the $p=2$ premise variables. For this, we again define

$$
\left\{\begin{array}{l}
F_{j, \text { min }}\left(z_{j}\right)=\frac{z_{j}-z_{j \min }}{z_{j \max } z_{j \min }} \\
F_{j, \max }\left(z_{j}\right)=\frac{z_{j \max }-z_{j}}{z_{j \max -} z_{j \min }}
\end{array}\right.
$$

Thus, the jth unmeasurable premise variable can be expressed as follows:

$$
z_{j}(t)=F_{j, \min }\left(z_{j}\right) z_{j \max }+F_{j, \max }\left(z_{j}\right) z_{j \min }
$$

Using this transformation, only $r=2^{2}$ submodels characterized by $p=2$ premise variables are generated. The TS model is then described by four fuzzy If-Then rules, where the $i^{t h}$ rule is of the following form:

Rule $R i$

$$
\begin{gathered}
\text { If }\left(z_{1}(t) \text { is } F_{1, k}\right) \text { and }\left(z_{2}(t) \text { is } F_{2, l}\right) \text { Then } \\
x(t)=A_{i} x(t)+B u(t)+D w(t) .
\end{gathered}
$$

With $k, l \in \min , \max \quad$ and $i=1,2,3,4$.

In the presence of sensor faults $f_{s}(t)$, the global fuzzy model can be transformed into the following state-space representation.

$\left\{\begin{array}{l}\dot{x}(t)=\sum_{i=1}^{4} h_{i}(\hat{x}(t))\left(A_{i} x(t)\right)+B u(t)+D w(t) \\ y(t)=C x(t)+D_{s} f_{s}(t)\end{array}\right.$

where

$$
\begin{aligned}
& \lambda_{i}\left(z_{1}(t), z_{2}(t)\right)=\prod_{k, l=1}^{2} F_{1}^{k}\left(z_{1}(t)\right) F_{2}^{l}\left(z_{2}(t)\right) \\
& h_{i}(z(t))=\frac{\lambda_{i}\left(z_{1}, z_{2}\right)}{\sum_{i=1}^{4} \lambda_{i}\left(z_{1}, z_{2}\right)} \\
& \sum_{i=1}^{4} h_{i}(z(t))=1
\end{aligned}
$$

Now, the idea is to express the TS models using weighting functions that depend on those estimates [15]. Thus, the TS model with measurable decision variables (10) is replaced with the following TS model, which involves only decision variables that are inaccessible for measurements:

$$
\left\{\begin{array}{l}
\dot{x}(t)=\sum_{i=1}^{4} h_{i}(\hat{x}(t))\left(A_{i} x(t)+B u(t)+D w(t)+x_{d}(t)\right) \\
y(t)=C x(t)+x_{s}(t)
\end{array}\right.
$$

where $x_{s}(t)=D_{s} f_{s}(t)$ corresponds to the effect of the faults on the outputs and $x_{d}(t)=\left(\sum_{i=1}^{8} h_{i}(x(t)) A_{i} x(t)-\sum_{i=1}^{4} h_{i}(\hat{x}(t)) A_{i} x(t)\right)$ represents the effect of the faults on the states.

In the following, this model is used to derive a descriptor observer that simultaneously estimates the state variables of the motor and the sensor faults.

\section{DESCRIPTOR OBSERVER DESIGN}

In this section, we propose a TS observer to simultaneously estimate the inaccessible states of rotor flux and the sensor faults. For this, an augmented system is constructed using the descriptor technique. Then the TS model (22) with unmeasurable decision variable and sensor faults can be written 
as the following TS system in descriptor form [16]:

$$
\left\{\begin{array}{l}
\bar{E} \dot{\bar{x}}=\sum_{i=1}^{4} h_{i}(\hat{x}(t)) \bar{A}_{i} \bar{x}(t)+\bar{B} u(t)+\bar{D}_{s} x_{s}(t)+\bar{D}_{w} w(t) \\
y(t)=\bar{C} \bar{x}(t)=C_{0} \bar{x}(t)+x_{s}(t)
\end{array}\right.
$$

where

$$
\begin{aligned}
& \bar{E}=\left(\begin{array}{lll}
I_{n} & 0_{n} & 0_{n \times p} \\
0_{n} & 0_{n} & 0_{n \times p} \\
0_{n} & 0_{n} & 0_{n \times p}
\end{array}\right), \bar{A}_{i}=\left(\begin{array}{ccc}
A_{i} & I_{n} & 0_{n \times p} \\
0_{n} & 0_{n} & 0_{n \times p} \\
0_{p \times n} & 0_{p \times n} & -I_{p}
\end{array}\right), \bar{x}(t)=\left(\begin{array}{c}
x(t) \\
x_{d}(t) \\
x_{s}(t)
\end{array}\right] \\
& , \bar{B}=\left(\begin{array}{c}
B \\
0_{n \times m} \\
0_{p \times m}
\end{array}\right), \bar{D}_{s}=\left(\begin{array}{c}
0 \\
0 \\
I_{p}
\end{array}\right), \bar{D}_{w}=\left(\begin{array}{c}
D \\
0 \\
0
\end{array}\right), \bar{C}=\left(\begin{array}{c}
C \\
0_{p \times n} \\
I_{p}
\end{array}\right), C_{0}=\left(\begin{array}{c}
C \\
0_{p \times n} \\
0_{p \times p}
\end{array}\right)
\end{aligned}
$$

Thus, the sensors faults $x_{s}(t)=D_{s} f_{s}(t)$ are considered as an auxiliary state of the augmented system (23). A descriptor observer is considered to estimate both the state variables of the motor and the sensor faults. For this, the following observer is proposed:

$$
\left\{\begin{array}{l}
E \dot{z}(t)=\sum_{i=1}^{r} h_{i}(z(t)) N_{i} z(t)+G_{i} u(t)+H_{i} w(t) \\
\hat{\bar{x}}(t)=z(t)+L y(t)
\end{array}\right.
$$

Where $z \in \square^{2 n+p}$ is the state of the observer and $\hat{\bar{x}} \in \square^{2 n+p}$ is the state estimation vector of the augmented system (23). In order to establish the conditions for the asymptotic convergence of the observer (24), we define the state estimation errors:

$$
\bar{e}(t)=\bar{x}(t)-\hat{\bar{x}}(t)
$$

The dynamics of the state estimation error can be evaluated by combining the equations (22) and (23) as follows:

$$
\begin{aligned}
& (\bar{E}+E L \bar{C}) \dot{\bar{x}}(t)-E \dot{\dot{\hat{x}}}(t)=\sum_{i=1}^{r} h_{i}(z(t))\left[\left(\bar{A}_{i}+N_{i} L C_{0}\right) \bar{x}(t)\right. \\
& \left.-N_{i} \hat{\bar{x}}(t)+\left(\bar{D}_{s}+N_{i} L\right) x_{s}(t)+\left(\bar{B}-G_{i}\right) u(t)+\left(\bar{D}_{w}-H_{i}\right) w(t)\right]
\end{aligned}
$$

If the observer matrices are selected as follows:

$$
\begin{aligned}
& E=\bar{E}+E L \bar{C} \\
& N_{i}=\bar{A}_{i}+N_{i} L C_{0} \\
& \bar{D}_{s}=-N_{i} L \\
& G_{i}=\bar{B} \\
& H_{i}=\bar{D}_{w}
\end{aligned}
$$

Then, the error dynamic (26) can be written as follows:

$$
E \dot{\bar{e}}=\sum_{i=1}^{r} h_{i}(z(t)) N_{i} \bar{e}(t)
$$

In order to satisfy the constraints (27), the observer parameters $N_{i}, L$ and $E$ can be selected as follows [11]:

$$
N_{i}=\left(\begin{array}{ccc}
A_{i} & I_{n} & 0_{n \times p} \\
0_{n} & 0_{n} & 0_{n \times p} \\
-C & 0_{p \times n} & -I_{p}
\end{array}\right)
$$

$$
\begin{aligned}
E & =\left(\begin{array}{ccc}
I_{n} & 0_{n} & 0_{n \times p} \\
0_{n} & I_{n} & 0_{n \times p} \\
Q C & 0_{p \times n} & Q
\end{array}\right) \\
L & =\left(\begin{array}{l}
0_{n} \\
0_{n} \\
I_{n}
\end{array}\right)
\end{aligned}
$$

$Q \in \square^{p \times p}$ is a full rank matrix that guarantees the non singularity of the matrix $E$, then the error dynamics (28) can be rewritten as follows:

$\dot{\bar{e}}(t)=\sum_{i=1}^{r} h_{i}(z(t)) S_{i} \bar{e}(t)$

with

$$
\begin{aligned}
S_{i} & =E^{-1} N_{i} \\
& =\left(\begin{array}{cc}
A_{d i} & 0 \\
-C_{d} A_{d i}-Q^{-1} C_{d} & -Q^{-1}
\end{array}\right)
\end{aligned}
$$

Avec

$$
A_{d i}=\left(\begin{array}{cc}
A_{i} & I_{n} \\
0 & 0
\end{array}\right), C_{d}=C 0
$$

The convergence condition of the state estimation error can be formulated by the following theorem.

\section{Theorem 1}

The state estimation error between the TS model (23) and its descriptor observer (24) converges asymptotically towards zero, if there exists a matrix $P=P^{T}>0$ and a non singular matrix $Q$ that verify the following condition:

$$
\bar{S}_{i}^{T} P+P \bar{S}_{i}<0 \quad \text { for } i=1,2,3,4
$$

As a result, sensor fault estimations can be obtained using the TS descriptor observer (20), from:

$$
\hat{f}_{s}(t)=D_{s}^{T} D_{s}^{-1} D_{s}^{T} x_{s}(t)
$$

with $D_{s}$ the matrix that gives the effect of the faults on the outputs, as defined in (10) and $x_{s}(t)=y(t)-C_{0} \bar{x}(t)$.

\section{SIMULATION RESULTS}

In this section, the efficiency of the proposed TS observer will now be demonstrated by using a simulation of a standard induction motor. This makes repeatable comparisons possible. 
The characteristics of the induction motor parameters correspond to an induction motor placed at the UCPI laboratory in Sfax, Tunisia [22]: Mutual inductance $M=447.5 \mathrm{mH}$, Moment of inertia $J=0.0293 \mathrm{Kg} . \mathrm{m}^{2}$, Stator resistance $R_{s}=10.5 \Omega$, Rotor resistance $R_{r}=4.3047 \Omega$, Stator inductance $L_{s}=471.8 \mathrm{mH}$, Rotor inductance $L_{r}=471.8 \mathrm{mH}$, and number of Pole Pairs $n_{p}=2$.

In all the results presented below, we have simulated a constant speed reference $\omega_{m}=100 \mathrm{rd} / \mathrm{s}$ and a load torque of $C_{r}=5 \mathrm{Nm}$, applied at time $t=1.5 \mathrm{sec}$. The full rank matrix $Q$ is chosen as $Q=\operatorname{diag} 0.001 \quad 0.001 \quad 0.001$. The premise variables are bounded as follows:

$$
\begin{aligned}
& \omega_{m_{\text {min }}}=-200(r d / s) ; \omega_{m_{\max }}=200(r d / s) \\
& i_{s d_{\min }}=-6 A ; \quad i_{s d_{\max }}=6 A ; i_{s q_{\min }}=-6 A ; i_{s q_{\max }}=6 A \\
& \Psi_{r d_{\min }}=-1.2 \mathrm{~Wb} ; \Psi_{r d_{\max }}=1.2 \mathrm{~Wb} ; \Psi_{r q_{\min }}=-1.2 \mathrm{~Wb} ; \Psi_{r q_{\max }}=1.2 \mathrm{~Wb}
\end{aligned}
$$

To test the proposed approach, a sequence of disconnections which affect the sensors current and the optical encoders is tested. More precisely, intermittent faults are injected successively into each of the three phases, (denoted by A, B, C) and in the rotor speed. The fault sequence diagram on each sensor is shown in Figure1.

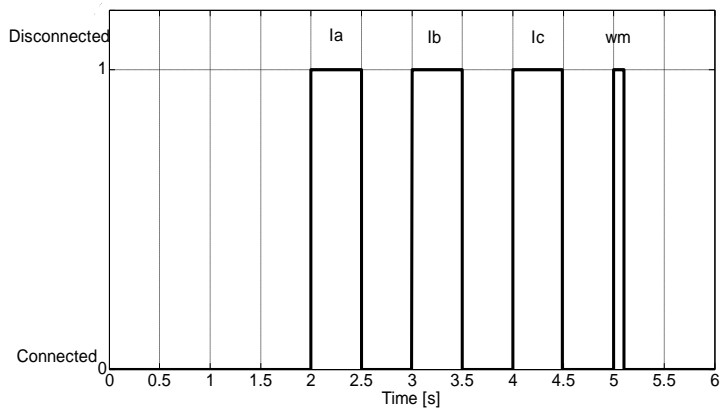

Figure 1. The sensor fault sequence diagram

The proposed observer is able to simultaneously estimate the system states $x(t)$, including the inaccessible variable rotor flux, and detect and isolate the sensor faults (See Figure 2).

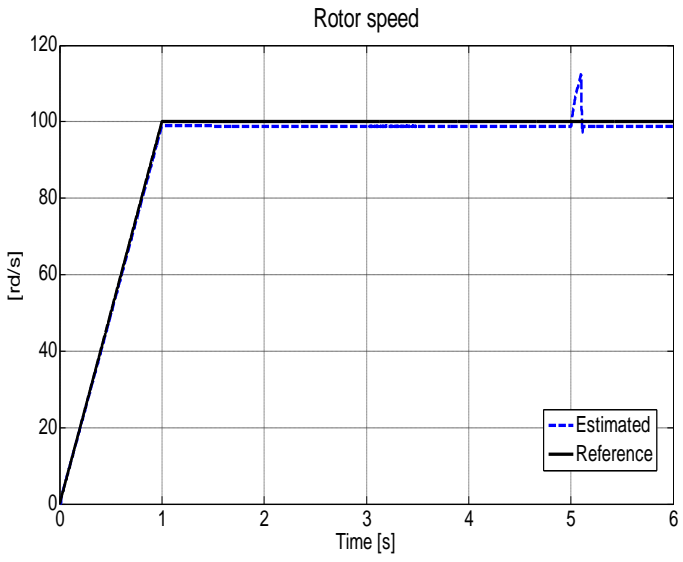



Figure 2. Rotor speed and rotor flux norm

Figure 3 shows the stator currents errors and the corresponding rotor speed errors estimated by the TS observer.
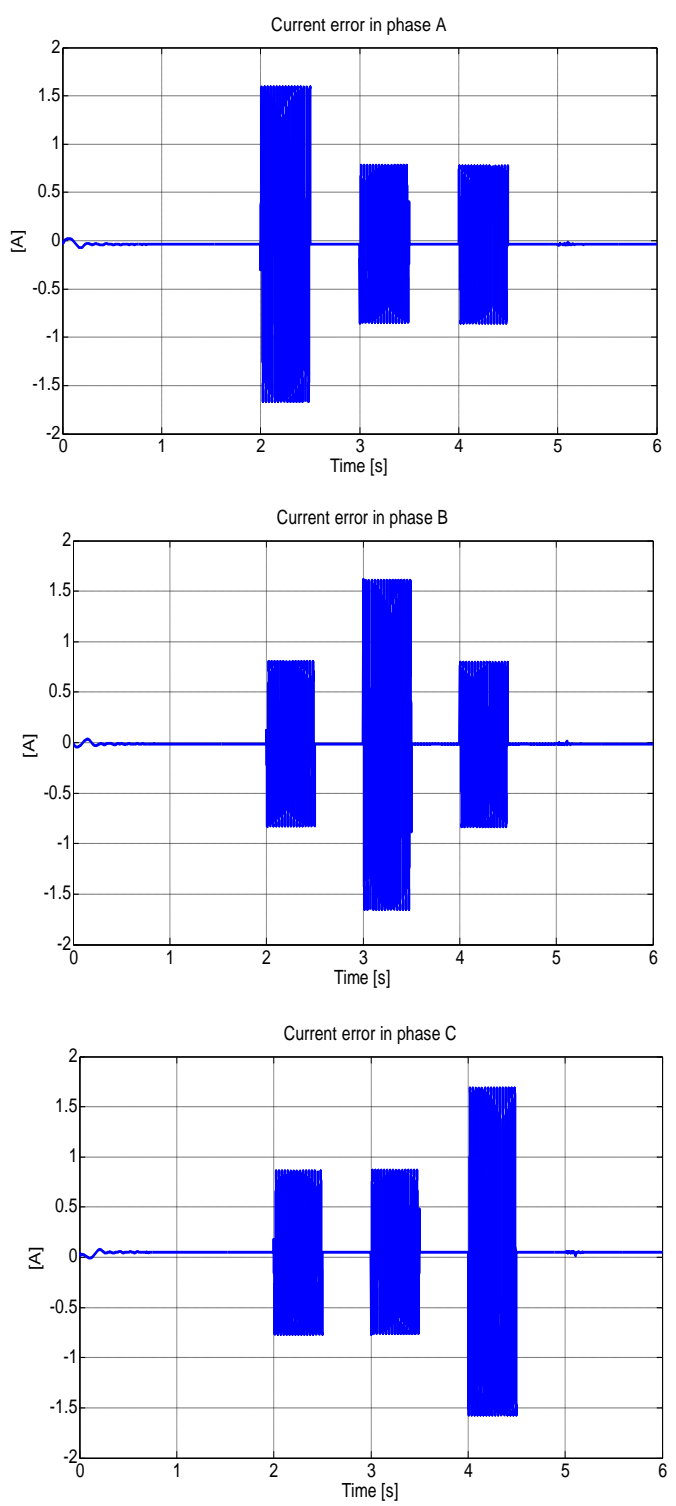


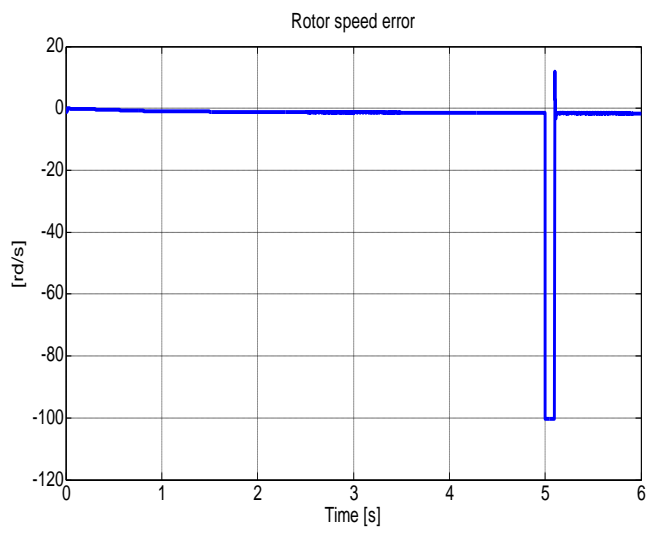

Figure 3. Stator currents and rotor speed errors

Once the magnitudes of the faults are estimated, using (33), a simple logic decision makes it possible to isolate the fault, as presented in Figure 4.

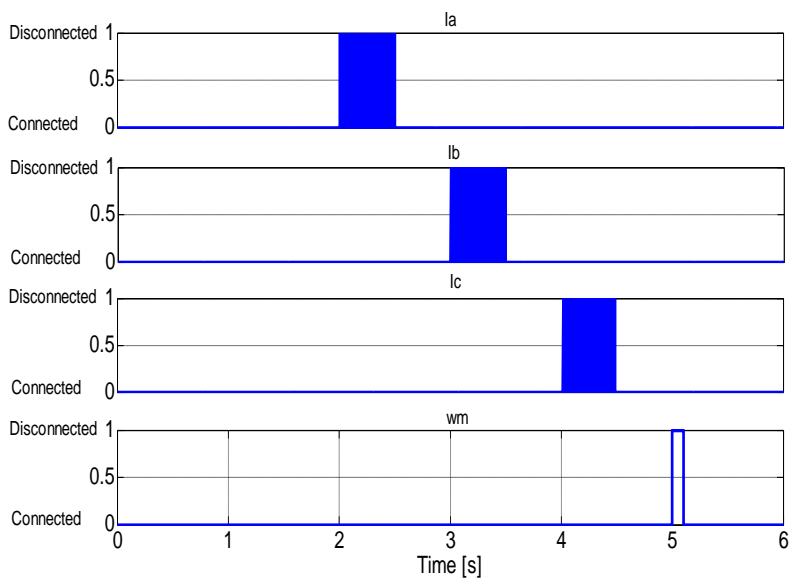

Figure 4. Detection of each sensor fault

Finally, and in order to show the influence of the measurable decision variables on the estimation quality of the fuzzy observer, a comparative study between the two structures of descriptor observer for the rotor speed response is presented. We consider always the case where the sensor is subjected to intermittent disconnections. Indeed, the use of a TS observer, based on the measurable decision variables in the control scheme, show that the rotor speed differs from the reference signal and may cause instability of the machine; whereas the chose of observer with unmeasurable decision variables gives only a small fluctuation on the rotor speed response when the faults appear, always remaining close to its reference value.

It should be noted that:

- For the descriptor observer design with measurable decision variables, the procedure for determine the observer parameters is similar to the one given by (27), just we consider $x_{d}(t)=0$ and take the same value of $Q$.
- The two frameworks of TS descriptor observers are affected by the same sequence of sensor disconnections and they are also submitted to the same input-output linearizing feedback control law.

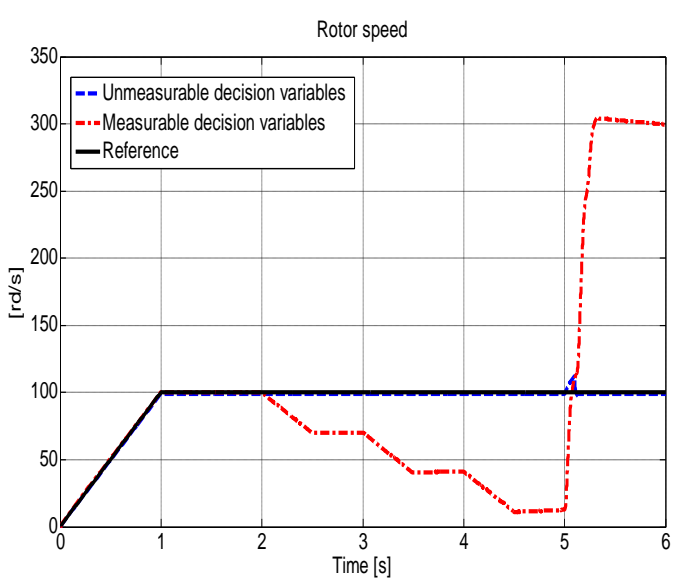

Figure 5. Comparison of the estimated rotor speed by the two structures of observers

\section{CONCLUSION}

In this study, an integral fuzzy control scheme for an induction motor via TS fuzzy model approach has been proposed. Based on the T-S fuzzy approach, a fuzzy ob-server-based tracking controller is designed to reduce the tracking error and to guaranty the disturbance rejection. The rotor flux is unavailable for measurement and it is estimated by a fuzzy observer. To solve the tracking control problem, an easy and systematic algorithm based on LMI optimization techniques is presented. The efficiency of the proposed controller is demonstrated through numerical simulations.

\section{REFERENCES}

[1] J. Zarei, J. Poshtan, "Bearing fault detection using wavelet packet transform of induction motor stator current". Tribology International 40 pp. 763-769, 2007.

[2] M.E.H. Benbouzid, "A review of induction motors signature analysis as a medium for faults detection". IEEE Trans. Ind. Electr. 47(5), pp. 984-93. 2000.

[3] M. Trabelsi, M .Boussak, M. Gossa, "Detection of shortcircuit's defect in the stator windings of the asynchronous motors by using the Park's vectors approach". 10th International conference on Sciences and Techniques of Automatic control \& computer engineering, December pp. 20-22, Hammamet, Tunisia. 2009.

[4] J. Faiz, B.M. Ebrahimi, H.A. Toliyat, B. Akin, Diagnosis of a mixed eccentricity fault in a squirrel-cage three-phase induction motor using time stepping finite element technique, in: Proc. IEEE IEMDC Conf., vol. 2, 2007, pp. 1446-1450.

[5] S. Bachir, S. Tnani, C.C. Trigeassou, G. Champenois, "Diagnosis by parameter estimation of stator and rotor 
occurring in induction machines", IEEE Trans. Ind. Electr. 53 (3) (2006) 963-973.

[6] S.M. Bennett, R.J. Patton, S. Daley, "Sensor fault-tolerant of a rail traction drive", Control Engineering Practice, 1999, vol. 7, pp. 217-225.

[7] CJL. Toribio, R.J. Patton, S. Daley, "Takagi-Sugeno Fuzzy Fault-Tolerant Control of an Induction Motor". Neural Computing \& Applications, Vol. 9, pp. 19-28, 2000.

[8] V.T. Tran, B.S. Yang, M.S. Oh, A.C.C. Tan, "Fault diagnosis of induction motor based on decision trees and adaptive neuro-fuzzy inference". Expert Systems with Applications 36, 2009 pp. 1840-1849

[9] P.V.J Rodriguez, A.Arkkio, "Detection of stator winding fault in induction motor using fuzzy logic". Applied Soft Computing Vol. 8, pp. 1112-1120, 2008.

[10] G.I.S. Palmero, J.J. Santamaria, E.J. M. Torre, J.R.P. Gonzalez, "Fault detection and fuzzy rule extraction in AC motors by a neuro-fuzzy ART-based system". Engineering Applications of Artificial Intelligence Vol. 18, pp. $867-$ 874, 2005.

[11] T. Czeslaw, K. Teresa, O. Kowalska, "Neural networks application for induction motor faults diagnosis". Mathematics and computers in simulation, 2003, 63, pp. 435-448.

[12] D. Pomorski, P.B. Perche, "Inductive learning of decision trees: application to fault isolation of an induction motor", Engineering Applications of Artificial Intelligence 14, 2001, pp. 155-166.

[13] T. Takagi, M. Sugeno, "Fuzzy identification of systems and its application to modeling and control", IEEE Transactions on Systems, Man and Cybernetics, 15(1):116-132, 1985.

[14] Y. Morère, "Mise en oeuvre de lois de commandes pour les modèles flous de type Takagi-Sugeno". PhD thesis from Université de Valenciennes et du Haut Cambrésis, Lille, France, January 4, 2001.

[15] D. Maquin, "State estimation and fault detection for systems described by Takagi-Sugeno nonlinear models", 10th International conference on Sciences and Techniques of Automatic control \& computer engineering, December 20-22, 2009, Hammamet, Tunisia.

[16] M. Bouattour, M. Chadli, A. El Hajjaji, M.Chaabane, "Sensor faults estimation for TS Models using descriptor techniques: Application to fault diagnostic", 7th IFAC Symposium SAFEPROCESS'09. 30 June-03 July 2009.

[17] R. Marino, S. Peresada, P. Valigi, "Adaptive input-output linearizing control of inductions motors", IEEE R.Marino, Transactions on automatic control, Vol 38, No.2, pp. 206219, 1993.

[18] P. Tomei, C.M. Verrelli. A global tracking control for speed-sensorless inductions motors. Automatica, Vol 40, pp. 1071-1077, 2004.

[19] D. Ichalal, B. Marx, J. Ragot, D. Maquin, State estimation of Takagi-Sugeno systems with unmeasurable premise variables, IET Control Theory Appl., 2010, Vol. 4, Iss. 5, pp. 897-908

[20] M. Nachidi, F. Rodríguez, F. Tadeo, J.L. Guzmán, TakagiSugeno Control Of Nocturnal Temperature In Greenhouses Using Air Heating, ISA Transactions, 50 (2011), pp. 315320

[21] K. Tanaka, H.O. Wang. Fuzzy control systems design and analysis: a linear matrix inequality approach. John Wiley \& Sons; 2001.

[22] M. Allouche, M. Chaabane, M. Soussi, D. Mehdi and A. Hajjaji, Takagi-Sugeno Fuzzy Sensor Faults Estimation of an induction motor, MED'10 Conference, Marrakech, Morocco, June 2010.

\section{AUTHORS PROFILE}

Moez ALLOUCHE received a Master degree from the National Engineering School of Sfax, Tunisia, in 2006. He also obtained the $\mathrm{Ph}$. D in Electrical Engineering from the National Engineering School of Sfax, Tunisia, in 2010. His current research interests are robust control, fuzzy logic control, Dstability analysis and applications of these techniques to induction motors.

Mohamed CHAABANE received a $\mathrm{PhD}$ in Electrical Engineering from the University of Nancy, France in 1991. He was associate professor at the University of Nancy and was a researcher at the Center of Automatic Control of Nancy (CRAN) from 1988-1992. He is currently a professor in ENI-Sfax and editor in chief of the International Journal on Sciences and Techniques of Automatic Control and Computer Engineering IJSTA (www.sta-tn.com). His main research interests are in the fields of robust control, time-delay systems, and descriptor systems.

Mansour SOUISSI received his $\mathrm{PhD}$ in Physics from the University of Tunis in 2002. He is Professor of Automatic Control at the Preparatory Institute of Engineers of Sfax, Tunisia. Since 2003, he has held a research position at the Automatic Control Unit, National School of Engineers of Sfax, Tunisia. His current research interests are robust control, optimal control, and fuzzy logic. Dr Souissi is a member of the organization committees of several national and international conferences (STA, CASA).

Driss MEHDI is currently a Professor at the Institute of Technology of Poitiers, France. He is the head of the research group "Multivariable Systems and Robust Control" in the Laboratory of Automatic and Industrial Informatics of Poitiers. His main fields of research include robust control, time-delay systems, robust root-clustering, descriptor systems, control of industrial processes, etc.

Fernando Tadeo is currently a Professor of Automatic Control in the School of Engineering of the University of Valladolid, Spain. His main fields of research include robust control, timedelay systems and nonlinear control. 